\title{
Heavy-quark physics with a tmQCD valence action
}

\section{F LPHA \\ Collaboration}

\section{A. Bussone ${ }^{* a, b}$, S. Chaves ${ }^{a}$, G. Herdoíza ${ }^{a, b}$, C. Pena ${ }^{a, b}$, D. Preti ${ }^{c}$, J.Á. Romero $^{b}$, J. Ugarrio ${ }^{a, b}$}

${ }^{a}$ Department of Theoretical Physics, Universidad Autónoma de Madrid, E-28049 Madrid, Spain

${ }^{b}$ Instituto de Física Teórica UAM-CSIC, c/ Nicolás Cabrera 13-15, Universidad Autónoma de

Madrid, E-28049 Madrid, Spain

${ }^{c} I N F N$, Sezione di Torino Via Pietro Giuria 1, I-10125 Turin, Italy

E-mail: andrea.bussone@uam.es

\begin{abstract}
We introduce a mixed-action approach based on CLS ensembles, where a valence $N_{f}=2+1+1$ Twisted Mass QCD action is combined with the $N_{f}=2+1$ non-perturbatively $\mathrm{O}(a)$-improved Wilson sea sector. We show that for maximally twisted valence quarks, the automatic $\mathrm{O}(a)$ improvement of this set-up holds up to lattice artifacts coming from sea quark mass effects. Furthermore, we introduce a three-dimensional Gradient Flow smearing in order to tame the signal to noise ratio problem.
\end{abstract}

The 36th Annual International Symposium on Lattice Field Theory

July 22-28, 2018

East Lansing, Michigan, USA

\footnotetext{
${ }^{*}$ Speaker.
} 


\section{Introduction}

Heavy-quark physics is particularly challenging on the Lattice since various energy scales have to be accommodated simultaneously. Finite volume corrections have to be negligible, resulting in the condition $m_{\pi} L \gg 1$, and small discretization errors are required, $a m_{h} \ll 1$, in order to have controlled cutoff effects. An obvious (but expensive) way to decrease discretization effects is to reduce the lattice spacing $a$, but for $a \lesssim 0.05 \mathrm{fm}$, simulations are plagued by the topological freezing problem. A complementary approach is to employ the Symanzik improvement programme [1], which allows to systematically remove $\mathrm{O}(a)$ effects in the observables of interest.

The CLS initiave has recently started a program of production of QCD configurations [9] with Lüscher-Weisz tree-level $\mathrm{O}\left(a^{2}\right)$-improved gauge action and $N_{f}=2+1$ non-perturbatively $\mathrm{O}(a)$ improved Wilson fermions. The use of (tree-level improved) open boundary conditions (open $\mathrm{BC}$ ) for the fundamental degrees of freedom is of paramount importance in reaching small lattice spacing while avoiding the topology freezing by allowing a smooth flow of the topological charge through the boundaries [8].

In addition to the reduction of the lattice spacing, it is also crucial to implement an improvement programme when aiming at reliable lattice QCD determinations of observables in the heavy-quark sector. In [2] it was elegantly explained how the $\mathrm{O}(a)$ effects are strictly connected to the hard breaking of chiral symmetry in the Wilson regularization and the rôle of the symmetries in the improvement programme was uncovered. Later, in [4], it was realized that physical observables are automatically $\mathrm{O}(a)$-improved in the Twisted Mass (TM) regularization [3], once the standard mass is set to its critical value.

The question we are addressing in this work is whether the use of TM only in the valence sector of the theory still retains the automatic $\mathrm{O}(a)$-improvement mechanism. The naïve expectation is that $\mathrm{O}(a)$ effects arising from the sea regularization could still contribute. In the first part of this work we will briefly review the argument of $\mathrm{O}(a)$ improvement for TM [4] and combine it with an extension of [6] to Wilson-like theories. ${ }^{1}$ The extension of the latter work, which was originally formulated only for Wilson fermions, is crucial in order to understand the contributions coming from sea and valence fermions to $\mathrm{O}(a)$ discretization effects. In the second part we will discuss the use of the Gradient Flow technique [7] restricted to an equal-time hyperplane in order to build smeared interpolating operators. Interpolating operators are important technical elements of heavy quark physics computations and the use of smearing helps in getting signals at earlier Euclidean times.

\section{2. $\mathrm{O}(a)$-improvement of Wilson-like regularizations}

We set up the infinite volume lattice theory in the Euclidean space. We restrict ourselves to an $\mathbf{S U}\left(N_{c}\right)$ gauge theory with $N_{f}$ non-degenerate quarks in the fundamental representation. Quarks and antiquarks $\psi, \bar{\psi}$ are taken as multiplets of $\mathbf{S} \mathbf{U}\left(N_{f}\right)$. We concentrate on the fermionic sector of the theory where the unimproved lattice action is given by

$$
\mathrm{S}[U, \bar{\psi}, \psi]=a^{4} \sum_{x} \bar{\psi}(x)\left[\frac{1}{2} \gamma_{\mu}\left(\nabla_{\mu}^{+}+\nabla_{\mu}^{-}\right)-\frac{a \mathbf{r}}{2} \nabla_{\mu}^{+} \nabla_{\mu}^{-}+\mathbf{m}_{\mathbf{0}}+i \boldsymbol{\mu} \gamma_{5}\right] \psi(x),
$$

with the standard and twisted bare quark-masses labeled by $\mathbf{m}_{\mathbf{0}}$ and $\boldsymbol{\mu}$, respectively, and $\mathbf{r}$ denotes

\footnotetext{
${ }^{1}$ Regularizations for which the limit of vanishing masses coincides with the Wilson regularization at zero mass.
} 
the Wilson parameter. ${ }^{2}$ In the following we will refer to the standard bare quark-mass as the subtracted one, given by $\mathbf{m}=\mathbf{m}_{\mathbf{0}}-m_{\mathrm{cr}} \mathbf{1}$. Throughout this work we will only consider diagonal standard masses, i.e., $\mathbf{m}=\operatorname{diag}\left(m_{1}, m_{2}, \ldots, m_{N_{f}}\right)$, and $\boldsymbol{\mu}=\mu_{a} T^{a}$ for TM, with $T^{a}$ generators of $\mathbf{S U}\left(N_{f}\right)$, while $\boldsymbol{\mu}=\mu_{0} \mathbf{1}+\mu_{a} T^{a}$ for the Osterwalder-Seiler (OS) case. Notice that we are working in the so-called twisted basis, and the contact with the Wilson formulation is easily established by setting the twisted-mass matrix to zero.

The discrete symmetries of the Wilson-like theory are the following $[4,5]$ :

- $R_{5} \times[\mathbf{m} \rightarrow-\mathbf{m}] \times[\mathbf{r} \rightarrow-\mathbf{r}] \times[\boldsymbol{\mu} \rightarrow-\boldsymbol{\mu}]$, where $R_{5}$ is given by ${ }^{3}$

$$
\psi(x) \stackrel{R_{5}}{\longrightarrow} \gamma_{5} \psi(x), \quad \bar{\psi}(x) \stackrel{R_{5}}{\longrightarrow}-\bar{\psi}(x) \gamma_{5} .
$$

- $R_{5} \times D$, where the $D$-transformation is given by

$$
\psi(x) \stackrel{D}{\rightarrow} \mathrm{e}^{i 3 \pi / 2} \psi(-x), \quad \bar{\psi}(x) \stackrel{D}{\rightarrow} \mathrm{e}^{i 3 \pi / 2} \bar{\psi}(-x), \quad U_{\mu}(x) \stackrel{D}{\rightarrow} U_{\mu}^{\dagger}(-x-\hat{\mu}) .
$$

- $P \times[\boldsymbol{\mu} \rightarrow-\boldsymbol{\mu}], T \times[\boldsymbol{\mu} \rightarrow-\boldsymbol{\mu}]$ and $C$, where $P, T$ and $C$ are respectively parity, time reversal and charge conjugation.

From the symmetries listed above one can infer that also $P_{5} \times[\mathbf{m} \rightarrow-\mathbf{m}] \times[\mathbf{r} \rightarrow-\mathbf{r}]$ is a symmetry of the action, where $P_{5}$ is given by the product $R_{5} \times P$.

Let us consider for the moment $N_{f}$ massless Wilson fermions. The theory possesses chiral symmetry and a convenient way to write down the improvement is given by, generalizing the notation in [6], $\operatorname{Tr}\left(T^{a} \Gamma \mathscr{O}\right) \equiv \bar{\psi} T^{a} \Gamma \psi=\operatorname{Tr}\left\{T^{a} \Gamma[\psi \otimes \bar{\psi}]\right\}$, where $\Gamma$ is an element of the Clifford algebra. Since we are considering Wilson-like theories the mass-independent improvement goes exactly as in $[2,6]$, while the massive one will be a slight modification of them. We give, as examples, the mass-dependent improvement of the Lagrangian masses and bilinears. In [6], in order to find the standard-mass dependence, a spurionic analysis was applied, by considering the standard-mass matrix to transform in the adjoint representation of $\mathbf{S} \mathbf{U}\left(N_{f}\right)$. We extend the spurionic analysis including the twisted-mass matrix, i.e., $i \gamma_{5} \boldsymbol{\mu} \rightarrow i \gamma_{5} U^{\dagger} \boldsymbol{\mu} U$. The operators contributing to the massive $\mathrm{O}(a)$ improvement are then found along the lines of [6].

\subsection{Lagrangian masses \& non-singlet bilinears}

The improvement of the diagonal Lagrangian-masses is found to be, taking into account the scalar (pseudoscalar) nature of the standard (twisted) masses, (see [6] for any unexplained notation)

$$
\begin{aligned}
& \widehat{m}_{j}=Z_{m}\{ {\left[m_{j}+\left(r_{m}-1\right) \frac{\operatorname{Tr} \mathbf{m}}{N_{f}}\right]+a\left[b_{m} m_{j}^{2}+\widetilde{b}_{m} \mu_{j}^{2}+\bar{b}_{m} m_{j} \operatorname{Tr} \mathbf{m}\right.} \\
&\left.\left.+\left(r_{m} d_{m}-b_{m}\right) \frac{\operatorname{Tr}\left(\mathbf{m}^{2}\right)}{N_{f}}+\left(r_{m} \bar{d}_{m}-\bar{b}_{m}\right) \frac{(\operatorname{Tr} \mathbf{m})^{2}}{N_{f}}+\left(r_{m} \widetilde{d}_{m}-\widetilde{b}_{m}\right) \frac{\operatorname{Tr}\left(\boldsymbol{\mu}^{2}\right)}{N_{f}}\right]\right\}, \\
& \widehat{\mu}_{j}=Z_{\mu} \mu_{j}\left[1+a\left(b_{\mu} m_{j}+\bar{b}_{\mu} \operatorname{Tr} \mathbf{m}\right)\right] .
\end{aligned}
$$

As another example of the improvement out of the chiral limit we give the expressions for nonsinglet bilinears

$$
\left.\widehat{\operatorname{Tr}} \widehat{\left(T^{a} \Gamma \mathscr{O}\right.}\right)=Z_{\mathscr{O}_{\Gamma}}\left\{\operatorname{Tr}\left(T^{a} \Gamma \mathscr{O}\right)^{I}\left[1+a \bar{b}_{\mathscr{O}_{\Gamma}} \operatorname{Tr} \mathbf{m}\right]+a \frac{b_{\mathscr{O}_{\Gamma}}}{2} \operatorname{Tr}\left(\left\{T^{a}, \mathbf{m}\right\} \Gamma \mathscr{O}\right)\right.
$$

\footnotetext{
${ }^{2}$ Boldface symbols are used for matrices in flavor space.

${ }^{3}$ In the continuum, $R_{5}$ is a symmetry of the integration measure for $N_{f}>1$ sea quarks [21]. When considering additional valence quarks, their contribution to the integration measure can be exactly suppressed through the inclusion of the corresponding ghost fields (see e.g. [5]).
} 


$$
\begin{aligned}
& +a f_{\mathscr{O}_{\Gamma}} \operatorname{Tr}\left(T^{a} \mathbf{m}\right) \operatorname{Tr}(\Gamma \mathscr{O})+a \widetilde{b}_{\mathscr{O}_{\Gamma \gamma_{5}}} \operatorname{Tr}\left(T^{a} i \boldsymbol{\mu} \Gamma \gamma_{5} \mathscr{O}\right) \\
& \left.+a \widetilde{f}_{\mathscr{C}_{\Gamma \gamma_{5}}} \operatorname{Tr}\left(T^{a} i \boldsymbol{\mu}\right) \operatorname{Tr}\left(\Gamma \gamma_{5} \mathscr{O}\right)+a \widetilde{\bar{b}}_{\mathscr{O} \gamma_{5}} \operatorname{Tr} i \boldsymbol{\mu} \operatorname{Tr}\left(T^{a} \Gamma \gamma_{5} \mathscr{O}\right)\right\} .
\end{aligned}
$$

Additional terms appear with respect to [6], some of which are absent in TM, while present in the OS case.

\subsection{On-shell automatic $\mathrm{O}(a)$-improvement}

In this section we recall the proof of automatic $\mathrm{O}(a)$-improvement given in [4]. Once the fermionic action and the bilinears are improved, one can be convinced that all the operators entering at $\mathrm{O}(a)$ in physical Green's functions are odd under sign exchange of the Wilson parameter. By considering the Wilson Averaging (WA) technique [4] and the symmetries given in Sect. 2 we obtain the improvement of Wilson-like theories. ${ }^{4}$ A higher power for $a^{n}$ corrections is found once the WA is applied on the $n$-point multiplicatively renormalizable correlation function $\Phi$,

$$
\frac{1}{2}[\langle\Phi\rangle|\underset{\substack{\mathbf{m} \\ \mathbf{m}}}{\mathbf{r}}+\langle\Phi\rangle| \underset{\substack{\mathbf{\mu} \\ \mathbf{r}}}{\mathbf{r}}]=\left\langle\Phi_{0}\right\rangle+\mathrm{O}\left(a^{2}\right) .
$$

Where the symbol $\left.\langle\ldots\rangle\right|_{\mathbf{r}, \ldots}$ refers to the expectation value calculated in the Lattice theory with parameters $\mathbf{r}, \ldots$, and $\Phi_{0}$ is the continuum counterpart of the lattice correlator $\Phi$. By using the $P_{5} \times[\mathbf{m} \rightarrow-\mathbf{m}] \times[\mathbf{r} \rightarrow-\mathbf{r}]$ symmetry one gets to the Mass Average (MA) [4]

$$
\frac{1}{2}\left[\langle\Phi\rangle\left|\underset{\substack{\mathbf{m} \\ \mathbf{r}}}{\mathbf{r}}+\eta^{P} \eta^{R_{5}}\langle\Phi\rangle\right| \underset{\substack{\mathbf{\mu} \\ \mathbf{r}}}{\mathbf{r}}\right]=\left\langle\Phi_{0}\right\rangle+\mathrm{O}\left(a^{2}\right),
$$

where we have denoted the parity and $R_{5}$-parity of the correlator with the corresponding $\eta$ 's. Now if we consider vanishing standard masses ${ }^{5}$ we obtain the automatic on-shell $\mathrm{O}(a)$-improvement for physical observables. In Fig. 1 we show the mechanism of $\mathrm{O}(a)$-improvement at tree-level in the
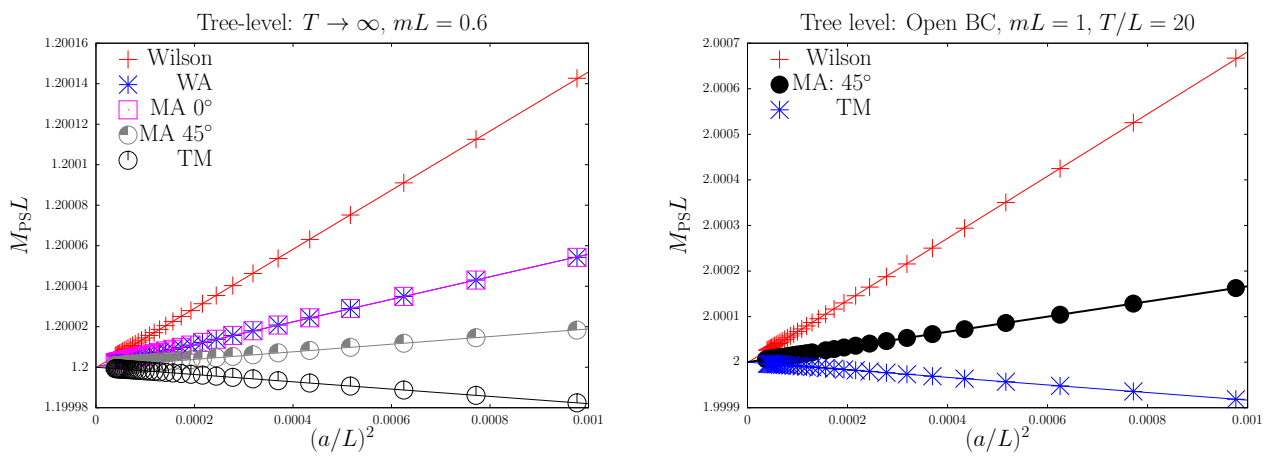

Figure 1: Left panel: Tree-level scaling of the mass-degenerate pseudoscalar meson mass in the infinite time extent limit. See [26] for a similar study. The Wilson data show $\mathrm{O}\left(a^{2}\right)$ scaling once the standard quark masses are improved. In the other cases no improvement of the masses is performed and the $\mathrm{O}\left(a^{2}\right)$ scaling is clearly visible. WA and MA (at vanishing angle) results are equivalent, since they are related by a symmetry. Right panel: Similar plot as in the left panel but in the open BC set-up. The formulae employed were taken from [11,22]. As before, the Wilson case has to be improved before getting the expected $\mathrm{O}\left(a^{2}\right)$ scaling.

\footnotetext{
${ }^{4}$ The inclusion of $c_{\mathrm{SW}}$ does not alter the conclusions in this section, the associated operator is odd under $r \rightarrow-r$.

${ }^{5}$ It is sufficient that the masses vanish up to $\mathrm{O}(a)$ effects.
} 
infinite time extent limit and with Dirichlet boundary conditions in the time direction. ${ }^{6}$

\section{Mixed action improvement}

We are now ready to set up the mixed action theory with Wilson fermions in the sea and TM in the valence sector. In [10] a self-adjoint, strictly positive transfer matrix was explicitly constructed for the the Wilson regularization $(r=1)^{7}$, and later on the proof was extended to the TM regularization $[11,12]$. Mixed actions are known to be non-unitary, ${ }^{8}$ since the valence determinant has to be suppressed by ghosts [24]. The symmetries of the valence action are easily extended to the ghost sector [5,23], but its inspection is not strictly necessary as long as we are interested in correlators made of valence quarks only. By using the valence WA and MA one discovers that $\mathrm{O}(a)$-effects proportional to the trace of the sea-quark mass matrix are not eliminated. As an example of these findings we give here the comparison between the quark-mass lattice artifacts in the Wilson, TM and mixed action specified above

$$
\begin{aligned}
\text { Wilson }: \widehat{m}_{i j} & =\frac{Z_{A}}{Z_{P}} m_{i j}\left[1+a\left(\widetilde{b}_{A}^{\prime}-\widetilde{b}_{P}^{\prime}\right) m_{i j}+a\left(\bar{b}_{A}^{\prime}-\bar{b}_{P}^{\prime}\right) \operatorname{Tr} \mathbf{m}\right]+\mathrm{O}\left(a^{2}\right), & & \text { [PCAC mass] } \\
\mathrm{TM}: \widehat{\mu}_{j} & =\frac{1}{Z_{P}} \mu_{j}+\mathrm{O}\left(a^{2}\right), & & \text { [Lagrangian mass] } \\
\text { Mixed action }: \widehat{\mu}_{j} & =\frac{1}{Z_{P}} \mu_{j}\left(1+a \bar{b}_{\mu} \operatorname{Tr} \mathbf{m}\right)+\mathrm{O}\left(a^{2}\right), & & \text { [Lagrangian mass] }
\end{aligned}
$$

where $\bar{b}_{A}^{\prime}=\bar{b}_{P}^{\prime}=\bar{b}_{\mu}=\mathrm{O}\left(g_{0}^{4}\right)$ start at two-loop in perturbation theory and $\widetilde{b}_{A}^{\prime}-\widetilde{b}_{P}^{\prime}=-0.0012 g_{0}^{2}+$ $\mathrm{O}\left(g_{0}^{4}\right)$ [25]. It is worth noting that the mixed action is free from valence $\mathrm{O}(a \mu)$ effects while cutoff effects proportional to the trace of the sea quark mass, $\operatorname{Tr} \mathbf{m}$, can contribute. Preliminary results on the charm mass scaling in $a$, obtained with the TM action in [15], are presented in [18].

\section{Smearing of interpolating operators}

A signal-to-noise ratio problem arises in the calculation of correlators when there are states contributing to the variance with energies smaller than twice the ground state energy. Its manifestation can be very severe, especially at large source-sink time separations, since the degradation of the signal is exponential $[19,20]$. A way to tame the problem is to employ an interpolating operator that has better overlap with the ground state, thus enabling the extraction of relevant quantities at earlier time separations. The smearing corresponds to employing non-local operators in space, that attempt to replicate the extended nature of hadronic states. The smearing of interpolating operators is a standard technique, with the Wuppertal smearing being one of the most prominent examples [13]. Here we introduce the Gradient Flow smearing [7] in an equal-time hyperplane (3D-GF). The flow equations are a coupled system of differential equations

$$
\left\{\begin{array} { l } 
{ \partial _ { t } B _ { j } ( t , x ) = D _ { k } G _ { k j } ( t , x ) } \\
{ \partial _ { t } \chi ( t , x ) = \nabla ^ { 2 } \chi ( t , x ) } \\
{ \partial _ { t } \overline { \chi } ( t , x ) = \overline { \chi } ( t , x ) \overleftarrow { \nabla ^ { 2 } } }
\end{array} \quad \text { with initial conditions: } \left\{\begin{array}{l}
\left.B_{j}(t, x)\right|_{t=0}=A_{j}(x) \\
\left.\chi(t, x)\right|_{t=0}=\psi(x) \\
\left.\bar{\chi}(t, x)\right|_{t=0}=\bar{\psi}(x)
\end{array}\right.\right.
$$

\footnotetext{
${ }^{6}$ The latter are the fermionic boundary conditions used in CLS open BC set-up.

${ }^{7}$ In [4] the theory with $r=-1$ was proven to possess site reflection positivity.

${ }^{8} \mathrm{~A}$ valence and sea matching, such that the correct continuum limit theory is obtained, can be performed, see $[16,17]$ for an example related to this work.
} 

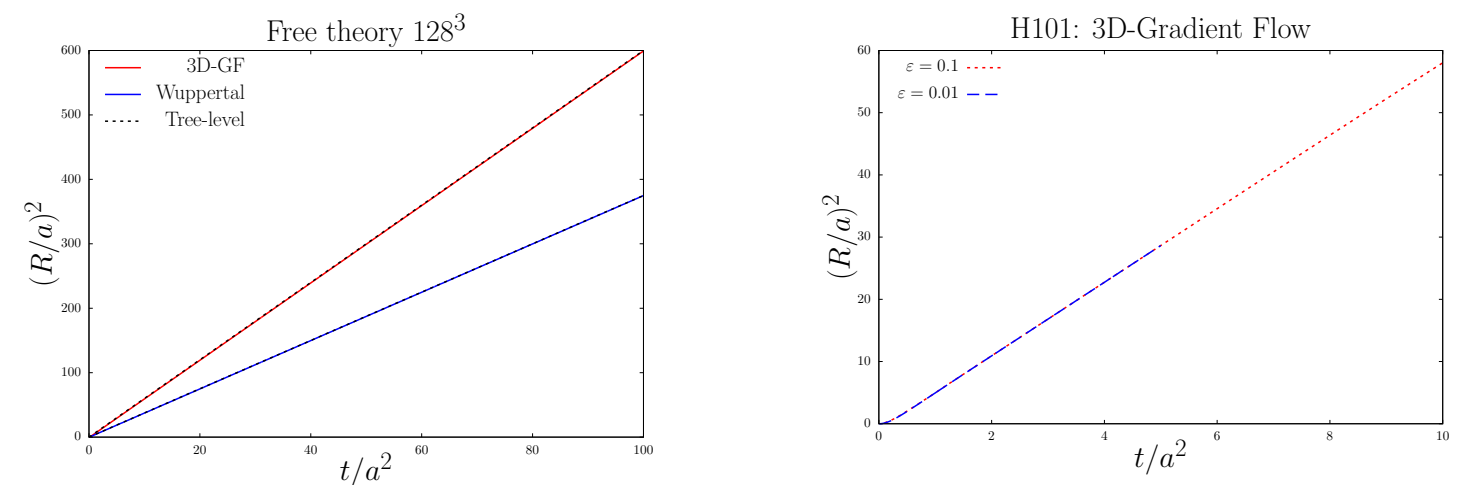

Figure 2: Left panel: smearing radius squared in the tree level theory as a function of the flow time. GF and Wuppertal agree with the theoretical expectations. Right panel: smearing radius squared of one configuration of the H101 ensemble $\left(m_{\pi}=m_{K}=420 \mathrm{MeV}, a=0.086 \mathrm{fm}\right)$ as a function of the flow time.

where $\nabla^{2}=D_{j} D_{j}$, with $D_{j}=\partial_{j}+B_{j}$. Note that compared to [7] the temporal components of the gauge field are not evolved. Furthermore, in contrast to Wuppertal smearing [13] the evolution of the fermionic and gauge degrees of freedom is done synchronously. The 3D-GF is still a Gaussian smearing with tree-level radius squared given by $R^{2}=2 D t$, and $D=3$. Upon discretization of the flow equations one can use different integrators [7]. In the case in which one fixes the background gauge field, for example (but not necessarily) at time $t=0$, the 3D-GF coincides with Wuppertal smearing, by further choosing the Euler integrator. In [7] the GF theory was proven to have good renormalization properties, in particular correlators of an arbitrary number of evolved gauge fields are finite and do not require further renormalization once the boundary (QCD) is renormalized. Naïvely the same can be expected in the 3D-GF framework if only correlators of gauge fields of type $B_{j}$ are considered, but further studies on the subject are required.

In [14] it was noted that no extra $\mathrm{O}(a)$-effects arise when considering the GF of a TM regularization with a degenerate doublet of quarks at maximal twist. A generalization of the arguments given in Sect. 2 allows to prove the absence of $\mathrm{O}(a)$ effects in both the GF and the 3D-GF when considering non-degenerate masses.

H101: 3D-Gradient Flow $\varepsilon=0.01, n=300$

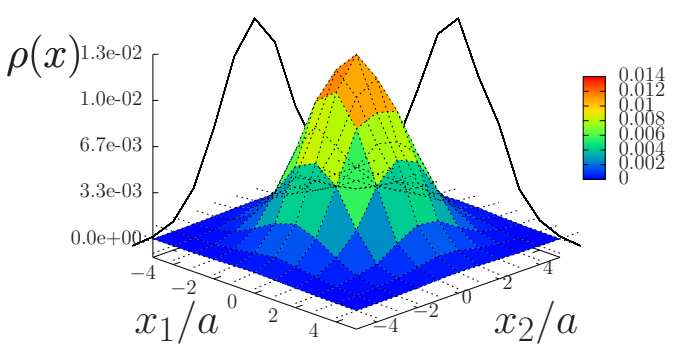

Figure 3: $\rho(x)$ defined as $\rho(t ; x)=|\chi(t ; x)|^{2} / \Sigma_{x}|\chi(t ; x)|^{2}$ at $t / a^{2}=3$, with $x_{0} / a=48$ and $x_{3} / a=0$.

\subsection{Tree-level results}

In the free theory, by starting from a $\delta$-function in position and color space, the smearing produces a Gaussian shape with radius squared $R^{2}$ given by: $6 t$ for 3D-GF and $6 t /(1+6 \varepsilon)$ for Wuppertal, where $t / a^{2}=n \varepsilon$, with $n$ iteration number and $\varepsilon$ step size. The results in the free theory are shown 
in the left panel of Fig. 2, where a perfect matching with the expectations is found. In the right panel of Fig. 2 we show the 3D-GF performed on one configuration of the H101 ensemble [9], with two different values of $\varepsilon$, which are in complete agreement. In Fig. 3 we show the result of the application of the 3D adjoint flow on a $\delta$-function source. The results are obtained through an extension of the code given in [27].

\section{Conclusions}

We have presented an analysis of the $\mathrm{O}(a)$-improvement by extending the work done in [6] to Wilson-like theories. We have analyzed the improvement in the case of a mixed action consisting of a TM regularization of the valence and a Wilson regularization of the sea sector. We have found that the automatic $\mathrm{O}(a)$-improvement is valid up to cutoff effects coming from sea quark masses and applied the Symanzik improvement programme to identify the relevant operators. Finally, we have presented the 3D Gradient Flow as a tool to fight the signal to noise ratio problem.

Acknowledgments: A.B. wishes to thank P. Dimopoulos and R. Frezzotti for useful discussions on the Twisted Mass regularization. We acknowledge PRACE for awarding us access to MareNostrum at the Barcelona Supercomputing Center (BSC), Spain. We acknowledge the use of the Hydra cluster at the Instituto de Física Teórica (IFT) were some part of the calculations were done. We acknowledge support through the Spanish MINECO project FPA2015-68541-P, the Centro de Excelencia Severo Ochoa Programme SEV-2016- 0597 and the Ramón y Cajal Programme RYC2012-10819. We are grateful to CLS members for producing the gauge configuration ensembles used in this study.

\section{References}

[1] K. Symanzik, Nucl. Phys. B 226 (1983) 187. doi:10.1016/0550-3213(83)90468-6

[2] M. Lüscher et al., Nucl. Phys. B 478 (1996) 365 doi:10.1016/0550-3213(96)00378-1

[3] R. Frezzotti et al. [Alpha Collaboration], JHEP 0108 (2001) 058

[4] R. Frezzotti and G. C. Rossi, JHEP 0408 (2004) 007 doi:10.1088/1126-6708/2004/08/007

[5] R. Frezzotti and G. C. Rossi, JHEP 0410 (2004) 070 doi:10.1088/1126-6708/2004/10/070

[6] T. Bhattacharya et al., Phys. Rev. D 73 (2006) 034504 doi:10.1103/PhysRevD.73.034504

[7] M. Lüscher, JHEP 1304 (2013) 123 doi:10.1007/JHEP04(2013)123

[8] M. Lüscher and S. Schaefer, JHEP 1107 (2011) 036 doi:10.1007/JHEP07(2011)036

[9] M. Bruno et al., JHEP 1502 (2015) 043 doi:10.1007/JHEP02(2015)043

[10] M. Lüscher, Commun. Math. Phys. 54 (1977) 283. doi:10.1007/BF01614090

[11] R. Frezzotti et al. [Alpha Collaboration], JHEP 0107 (2001) 048 doi:10.1088/1126-6708/2001/07/048

[12] A. Shindler, Phys. Rept. 461 (2008) 37 doi:10.1016/j.physrep.2008.03.001

[13] C. Alexandrou et al., Phys. Lett. B 256 (1991) 60. doi:10.1016/0370-2693(91)90219-G

[14] A. Shindler, Nucl. Phys. B 881 (2014) 71 doi:10.1016/j.nuclphysb.2014.01.022

[15] C. Pena, S. Sint and A. Vladikas, JHEP 0409 (2004) 069 doi:10.1088/1126-6708/2004/09/069

[16] G. Herdoíza et al., EPJ Web Conf. 175 (2018) 13018 doi:10.1051/epjconf/201817513018

[17] A. Bussone, G. Herdoíza, C. Pena, D. Preti, J.Á. Romero, J. Ugarrio, PoS LATTICE 2018 (2018) 318

[18] A. Bussone, G. Herdoíza, C. Pena, D. Preti, J.Á. Romero, J. Ugarrio, PoS LATTICE 2018 (2018) 271

[19] G. Parisi, Phys. Rept. 103 (1984) 203. doi:10.1016/0370-1573(84)90081-4

[20] G. P. Lepage, CLNS-89-971.

[21] S. Sint, Nucl. Phys. B 847 (2011) 491 doi:10.1016/j.nuclphysb.2011.02.002

[22] M. Lüscher and P. Weisz, Nucl. Phys. B 479 (1996) 429 doi:10.1016/0550-3213(96)00448-8

[23] M. Golterman et al., Phys. Rev. D 71 (2005) 094503 doi:10.1103/PhysRevD.71.094503

[24] A. Morel, J. Phys. (France) 48 (1987) 1111. doi:10.1051/jphys:019870048070111100

[25] Y. Taniguchi and A. Ukawa, Phys. Rev. D 58 (1998) 114503 doi:10.1103/PhysRevD.58.114503

[26] K. Cichy et al., Nucl. Phys. B 800 (2008) 94 doi:10.1016/j.nuclphysb.2008.03.004

[27] M. Lüscher and S. Schaefer, Comput. Phys. Commun. 184 (2013) 519 doi:10.1016/j.cpc.2012.10.003 